\title{
Compact water-window transmission X-ray microscopy
}

\author{
M. BERGLUND*, L. RYMELL*, M. PEUKER $\dagger$, T. WILHEIN $\dagger \&$ H. M. HERTZ* \\ *Biomedical and X-Ray Physics, Royal Institute of Technology, SE-10044 Stockholm, Sweden \\ †Forschungseinrichtung Röntgenphysik, Georg-August Universität, D-37073 Göttingen, Germany
}

Key words. Compact, droplet target, laser plasma, multilayer optics, soft X-ray microscopy, water window, zone plate optics.

\begin{abstract}
Summary
We demonstrate sub-100 nm resolution water-window soft $X$-ray full-field transmission microscopy with a compact system. The microscope operates at $\lambda=3.37 \mathrm{~nm}$ and is based on a $100 \mathrm{~Hz}$ table-top regenerative debris-free droplettarget laser-plasma X-ray source in combination with normal-incidence multilayer condenser optics for sample illumination. High-spatial-resolution imaging is performed with a $7.3 \%$ efficiency nickel zone plate and a $1024 \times 1024$ pixel CCD detector. Images of dry test samples are recorded with exposure times of a few minutes and show features smaller than $60 \mathrm{~nm}$.
\end{abstract}

\section{Introduction}

New high-resolution imaging techniques have historically led to important advances in many scientific fields, including biology. However, studies of unprepared samples in their natural state are still primarily performed with optical microscopy, where resolution is limited to approx. $250 \mathrm{~nm}$. Higher-resolution techniques (for example electron microscopy and scanned probe microscopies) either require significant sample preparation and/or are not easily adaptable to thick objects. X-ray microscopy in the waterwindow region $(\lambda=2.3-4.4 \mathrm{~nm})$ is an attractive technique for high-spatial-resolution imaging due to the possibility to study hydrated unstained, e.g. biological, samples that are several micrometres thick (Schmahl et al., 1993; Kirz et al., 1995). The few full-field transmission X-ray microscopes that are currently in operation rely on high-brightness synchrotron-radiation sources in order to achieve short exposure times. In this paper we present a table-top soft Xray full-field transmission microscope based on a droplettarget laser-plasma source. To our knowledge this is the first compact water-window transmission X-ray microscope demonstrating reproducible subvisible resolution imaging with good signal-to-noise ratio. Such compact instruments

Correspondence: H. M. Hertz. Tel: +46 8790 6216; fax: + 468205 609; e-mail: hertz@physics.kth.se show promise for increased scientific impact, as they allow the spread of X-ray microscopy to the small-scale laboratory.

Current transmission X-ray microscopes utilize the natural contrast between carbon-based substances (e.g. protein) and water in the water-window (Fig. 1). A typical arrangement includes a synchrotron-radiation source which illuminates the sample with a diffractive condenser zone plate (Schmahl et al., 1993). A high-resolution micro zone plate (MZP) acts as an objective. The magnified image can be directly read out from a thinned back-illuminated charge-coupled device (CCD) array. Synchrotron-based microscopes have found applications in, e.g. studies of malaria (Magowan et al., 1997), sperms (Abraham-Peskir et al., 1998), cytoskeletal elements (Scherfeld et al., 1998) and soils (Thieme et al., 1998). The resolution is $30-50 \mathrm{~nm}$ and the exposure time typically a few to $10 \mathrm{~s}$. With cryogenic sample preparation, the effect of radiation damage is reduced by several orders of magnitude (Schneider, 1998). Scanning microscopes, which are based on the same MZP optics, result in less radiation damage but longer exposure times. Such microscopes have also demonstrated biochemical imaging by, e.g. exploiting the chemical specificity of near-edge X-ray absorption resonances (Boese et al., 1997). Labelling techniques based on colloidal gold (Chapman et al., 1996) and X-ray-excited visible luminescence (Moronne, 1999) show promise for biochemical selectivity similar to optical fluorescence microscopy but with much higher spatial resolution. In addition to these synchrotron-based methods, non-compact X-ray microscopy has been demonstrated with a single-shot X-ray laser source outside the water window (Da Silva et al., 1992).

Non-synchrotron-based microscopes employ compact laser-plasma or pinch-plasma sources. Outside the water window, several compact transmission and scanning microscopes based on X-ray mirrors have been developed for longer wavelengths (10-20 nm) (Artioukov et al., 1995). These wavelengths are, however, of less interest for imaging of wet specimens. Water-window systems with mirror optics (e.g. Wolter-type objectives) have a larger collection efficiency 
than the zone-plate systems discussed below but generally exhibit a resolution much lower than optical microscopes (Aoki et al., 1998). Lensless imaging with laser-plasma sources is performed with contact microscopy, although this technique involves a more complicated development and read-out process, and it is difficult to obtain high resolution for thick samples (Stead et al., 1988).

Inside the water window, attempts at compact highresolution transmission microscopy have been based on zone-plate optics and laser-plasma or pinch-plasma sources. A solid-carbon-target laser plasma has been combined with an elliptical condenser and a zone plate objective to perform imaging of dry test objects (Nakayama et al., 1994). The magnification was $286 \times$ and the resolution slightly better than in an optical microscope. The debris emission from and non-regenerative character of the carbon target results in limited operability of the system. A low-repetition-rate pinch-plasma source has been combined with elliptical condenser and zone plate optics (Rudolph et al., 1994) for wet and dry imaging. The low repetition rate and instability of the source makes the system less operative, although 100-150 nm features were detectable on dry objects with low signal-to-noise ratio. For both sources the somewhat large line width (typically $\lambda / \Delta \lambda$ 100-300), producing chromatic aberrations in the diffractive zone-plate optics, results in limited extendability towards very high-resolution $\mathrm{X}$-ray microscopy.

In the present paper we demonstrate that high-spatialresolution (sub-100 nm) water-window full-field transmission X-ray microscopy can be performed with reasonable exposure times and good contrast with a table-top arrangement employing zone-plate imaging. The arrangement is described in the next section and is based on a droplet-target laser plasma source. It allows long-term operation due to the negligible debris emission from the source, high average power due to the regenerative target type, and has the narrow line width allowing extension to very-high-resolution imaging. Furthermore, with the use of a normal-incidence spherical condenser instead of grazing-incidence mirrors, the system is easy to align. We demonstrate the performance of the system on dry samples.

\section{Experimental arrangement}

The experimental arrangement for the compact X-ray microscope is shown in Fig. 2. The arrangement includes a regenerative droplet-target laser-plasma X-ray source, a normal-incidence multilayer condenser mirror, a sample, a zone plate for high-resolution imaging and a backilluminated soft X-ray sensitive CCD detector.

The laser-produced plasma is well known as a compact high-brightness X-ray source. Unfortunately, with conventional solid targets, debris emission will damage fragile $\mathrm{X}$ ray optics (such as zone plates or thin filter foils). With the use of microscopic liquid droplets (Rymell \& Hertz, 1993; Berglund et al., 1996; Hertz et al., 1998) as target, the debris emission is reduced several orders of magnitude compared to conventional targets (Rymell \& Hertz, 1995) and is completely eliminated for certain liquids (Rymell et al., 1995). In a typical arrangement, laser pulses from a $10 \mathrm{~Hz}$, 100 ps frequency-doubled Nd:YAG laser are focused onto $\sim 15 \mu \mathrm{m}$ diameter ethanol droplets, which are generated by a piezoelectrically vibrated glass capillary nozzle. Figure 1 shows the water-window spectrum, consisting of narrowbandwidth line-emission of hydrogen and helium-like carbon and oxygen in the water-window. Figure 1 also depicts the
Fig. 1. Absorption of protein and water in the water-window (logarithmic scale, left) and emission spectrum from ethanol droplettarget laser plasma (linear scale, right). The arrow indicates the $\lambda=3.37 \mathrm{~nm}$ line used for the microscopy.

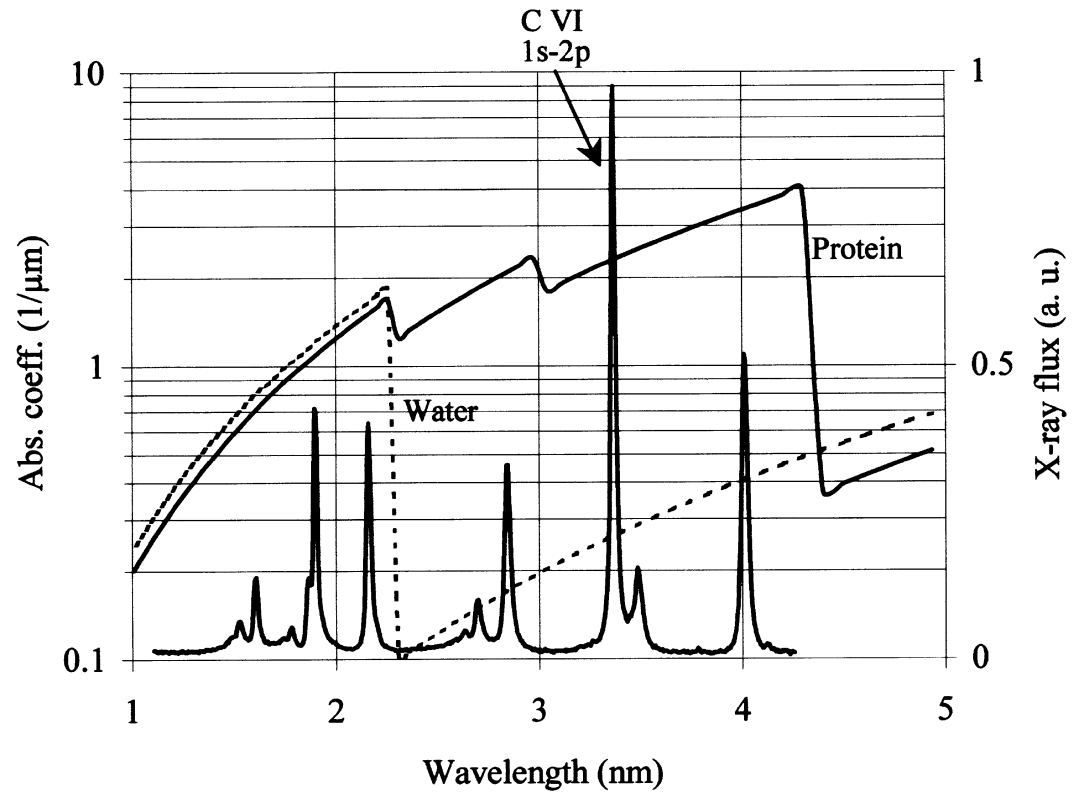




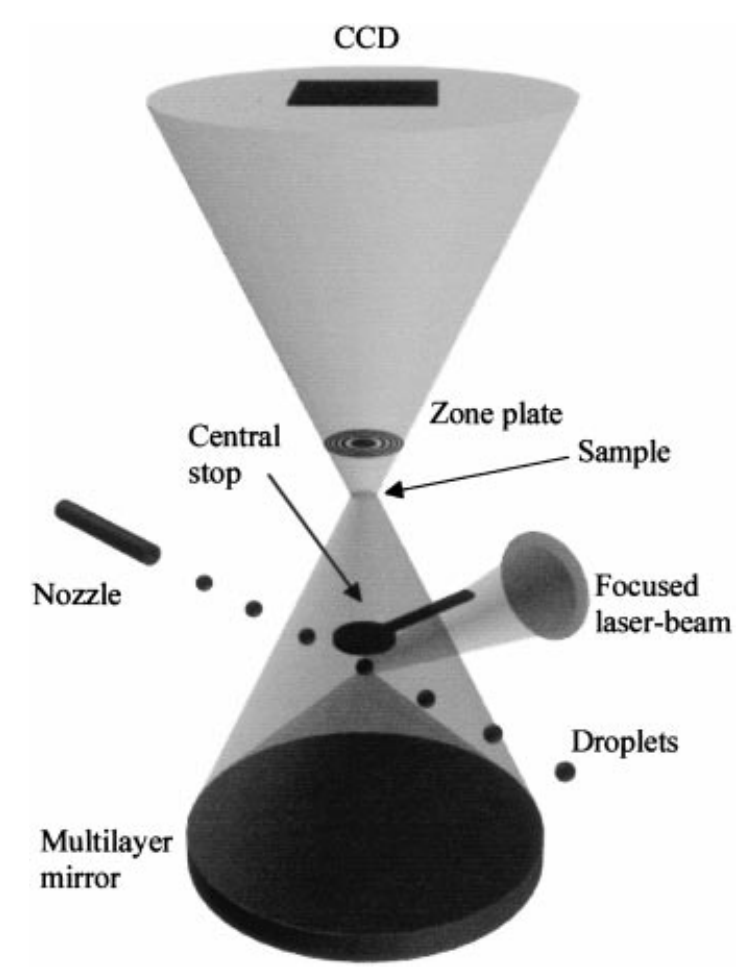

Fig. 2. Table-top water-window X-ray microscopy arrangement.

absorption of protein and water in the same wavelength range, showing the natural contrast.

Another advantage of the droplet-target system is its regenerative nature. It produces target material at a high rate, allowing full-day operation without interrupts and high-repetition-rate lasers to be employed. Thus, the high average power important for short exposure times may be obtained by using high-repetition-rate lasers. In order to reduce exposure times in the present experiment we used a $100 \mathrm{~Hz}$ frequency-doubled, $3 \mathrm{~ns}, 100 \mathrm{~mJ} /$ pulse Nd:YAGlaser (Coherent Infinity). Ethanol was used as target liquid. The emission spectrum is the same as in Fig. 1. The flux was typically $\sim 10^{12}$ photons $/($ sr $\times$ line $\times$ pulse $)$ and the source diameter (FWHM) was typically $\sim 25 \mu \mathrm{m}$. The microscopy is performed with the $\lambda=3.37 \mathrm{~nm}$ line from the $1 \mathrm{~s}-2 \mathrm{p}$ transition in C VI, as indicated by the arrow in Fig. 1. In previous measurements the bandwidth of this line has been determined to $\lambda / \Delta \lambda>500$ (Wilhein et al., 1997), allowing high-resolution imaging with diffractive zone plates.

Because the source emits into $4 \pi$ steradians, highcollection-efficiency condenser optics are necessary to obtain sufficient photon density in the object plane for reasonable exposure times in microscopy. Furthermore, the condenser should match the numerical aperture (NA) of the imaging optic (the zone plate) to allow high-resolution imaging. Previously, compact sources have been combined with elliptical optics (Nakayama et al., 1994; Rudolph et al., 1994), toriodal optics (Aoki et al., 1998), and diffractive optics (Schmahl et al., 1992).
We employ a normal-incidence water-window spherical multilayer condenser mirror (Hertz et al., 1999). This condenser arrangement has the advantage of intrinsic selection of a single line from the emitted multi-line laser-plasma radiation. This is necessary for high-resolution imaging with the diffractive zoneplate optics. Furthermore, it allows for a compact arrangement, and alignment is easy, as aberrations are much smaller than for, e.g. elliptical optics. Finally, this emerging mirror technology shows promise for large improvements as regards reflectivity, and thus exposure times.

The $58 \mathrm{~mm}$ diameter mirror has a radius of curvature of $343 \mathrm{~mm}$. Two hundred bilayers of $\mathrm{W} / \mathrm{B}_{4} \mathrm{C}$ were magnetron sputtered with a $2 \mathrm{~d}$-spacing corresponding to first-order normal-incidence reflectivity at $\lambda=3.37 \mathrm{~nm}$ resulting in an average reflectivity of $\sim 0.5 \%$. The narrow bandwidth (experimentally determined to $\lambda / \Delta \lambda \approx 80$ ) selects the line at $3.37 \mathrm{~nm}$ from the plasma, thereby reducing the image degradation due to lines at other wavelengths to negligible levels. The mirror is positioned approximately $263 \mathrm{~mm}$ below the source, resulting in an NA which corresponds to a $30 \mathrm{~nm}$-resolution zone plate. The source is magnified $1.8 \times$, theoretically producing a $\sim 45 \mu \mathrm{m}$ diameter (FWHM) illuminated spot in the object plane. A central stop blocks the direct light from the plasma. Furthermore, it eliminates the zeroorder radiation from the zone-plate, thereby creating a $25-\mu \mathrm{m}$ diameter image field (at $1000 \times$ magnification). A 350-nm free-standing titanium filter blocks scattered and reflected visible laser light from reaching the detector.

In the current arrangement the specimen is placed on a $50 \mathrm{~nm}$ thick $(500 \times 500 \mu \mathrm{m}) \mathrm{Si}_{3} \mathrm{~N}_{4}$ foil. The foil is inserted in a mechanical assembly which includes the zone plate. Horizontal and vertical positioning of the zone plate with respect to the sample is performed under a $400 \times$ inverted optical microscope. The microscope is equipped with a nanometre-gauge (Heidenhain) for accurate adjustment of the zone plate-specimen distance before the sample/zone plate assembly is inserted in the X-ray microscope.

High-resolution imaging is performed with a $56.1 \mu \mathrm{m}$ diameter nickel phase zone plate having 468 zones and an outermost zone width of $30 \mathrm{~nm}$. The focal length is $498 \mu \mathrm{m}$ at $\lambda=3.37 \mathrm{~nm}$. This high aspect ratio zone plate (mean aspect ratio $4.7: 1$ ) was fabricated utilizing a tri-level nanostructuring process. The pattern is generated by e-beam lithography and transferred by reactive ion etching (RIE) and Ni electrodeposition (Schneider et al., 1995; Peuker et al., 1998). The first-order efficiency has been measured at the BESSY synchrotron radiation source to $10.1 \%$ at $\lambda=2.4 \mathrm{~nm}$, resulting in an estimated efficiency at $3.37 \mathrm{~nm}$ of $7.3 \%$. The image is detected on a cooled, thinned, backilluminated $1024 \times 1024$ pixel CCD array (Photometrics) with $24 \times 24 \mu \mathrm{m}$ pixels and a quantum efficiency of $>0.6$. By adjusting the distance between the detector and the zone plate, the magnification was varied between $650 \times$ and $1000 \times$. 
a)

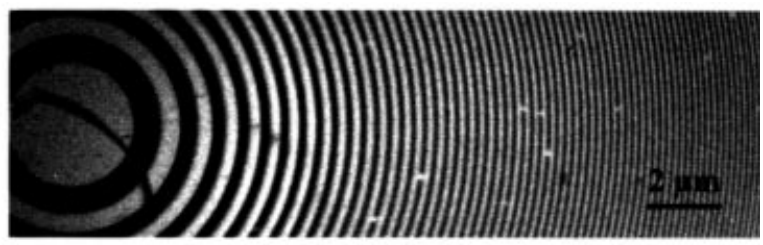

b)

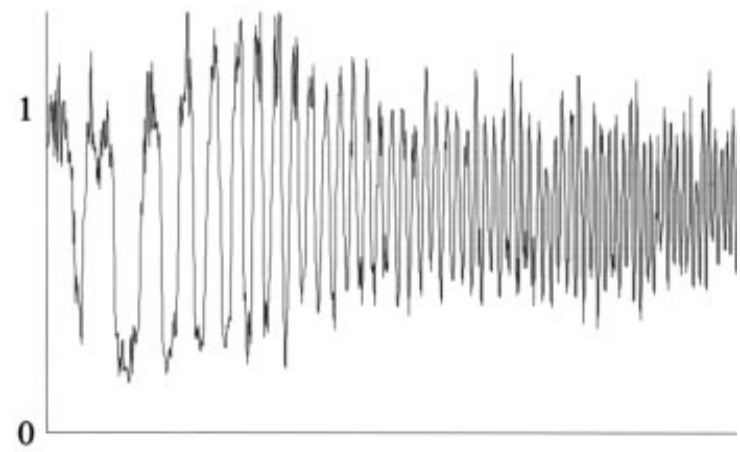

c)

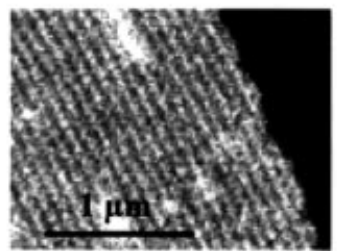

Fig. 3. Test image of zone-plate pattern (a) showing sub-60 nm features at outer zones (c). Magnification is $1000 \times$. (b) the intensity at a radial cross-section through (a).

Figure 3 shows a test image of a gold zone plate with $1000 \times$ magnification. This $70 \mu \mathrm{m}$ diameter zone plate has an outer zone width of $50 \mathrm{~nm}$. It is prepared with $200 \mathrm{~nm}$ $\mathrm{Au}$ on a $180 \mathrm{~nm} \mathrm{Si} \mathrm{substrate.} \mathrm{The} \mathrm{exposure} \mathrm{time} \mathrm{was}$ $\sim 2$ min. Figure 3(a) shows the inner zones and the curve in Fig. 3(b) shows the intensity at the radial cross-section of Fig. 3(a), indicating good contrast. Figure 3(c) shows the outer zones of the test object. The smallest zones in the image field are $58 \mathrm{~nm}$, which are clearly resolved.

In Fig. 4 a diatom is imaged with $650 \times$ magnification. The exposure time was $2 \mathrm{~min}$. Structures down to $<100 \mathrm{~nm}$ are observable. Comparison with optical microscopy shows that the fine-line gratings are only visible in the X-ray images.

\section{Discussion}

We have demonstrated a compact arrangement that allows high-resolution water-window full-field transmission soft Xray microscopy to be performed with reasonable exposure times and good signal-to-noise ratio. However, this first arrangement may be improved in several respects, especially X-ray flux (i.e. exposure time), resolution and operating wavelength. This will be discussed below.

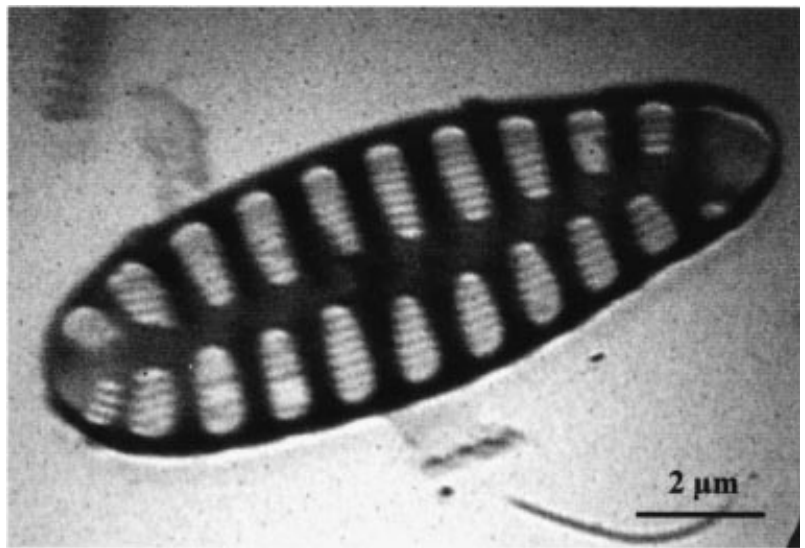

Fig. 4. Image of diatom with $650 \times$ magnification.

In the current microscope arrangement we typically record 160 photons $/($ pixel $\times$ min) at the CCD with $1000 \times$ magnification. Thus, approximately $6 \mathrm{~min}$ are required to obtain high-quality images (1000 photons/pixel). Such images are currently recorded with exposure times of a few seconds at synchrotron-based microscopes.

The current photon number (and thus exposure time) is consistent with our data regarding source brightness, laser repetition rate, condenser mirror reflectivity and surface waviness, Ti filter and $\mathrm{Si}_{3} \mathrm{~N}_{4}$ foil absorption, zone-plate efficiency, and CCD efficiency. The major necessary improvement to decrease our exposure times is improvement of the condenser mirror, both with regards to reflectivity and surface waviness (Hertz et al., 1999). Improving the reflectivity from the current approx. $0.5 \%$ to 3\% (Salashchenko et al., 1995) and reducing the surface waviness so that the full X-ray flux is imaged geometrically, would increase object plane flux $25-50 \times$. This would result in exposure times on the order of $10 \mathrm{~s}$ with synchrotron-quality images. Furthermore, improvements in laser technology are making $1000 \mathrm{~Hz}$ lasers commercially available. This would reduce the exposure time to a few seconds, thereby making table-top $\mathrm{X}$-ray microscopy competitive with current synchrotronbased microscopes.

The exposure time in the current arrangement is limited to approximately $5 \mathrm{~min}$ due to thermal drifts in the mechanical arrangement. Owing to this time limitation high-quality imaging may be performed down to a pixel size of $\sim 25 \mathrm{~nm} \quad(1000 \times$ magnification $)$. We are currently designing an improved system, including a wet cell, that will allow us to reach a zone-plate-limited resolution.

The current microscope operates at $\lambda=3.37 \mathrm{~nm}$. This wavelength exhibits a considerably larger absorption in carbon-based substances than wavelengths in the lower part of the water window, making imaging of thick ( $>$ a few micrometres) samples difficult. Initial attempts to image red blood cells and fixed renal epithelial cell line LLCPK reveal this problem, resulting in little detail in thick parts of the 
samples due to low transmitted photon flux. Therefore, the operating wavelength of the microscope should preferably be in the lower part of the water window. Compact liquid-jet laser-plasma sources for lower-wavelength emission have been developed. These are based on nitrogen ion emission at $\lambda=2.48$ and $\lambda=2.88 \mathrm{~nm}$ (Rymell et al., 1995; Berglund et al., 1998). Efforts are underway to adapt the condenser mirror to the smaller multilayer d-spacing necessary for these shorter wavelengths.

\section{Acknowledgements}

The authors gratefully acknowledge G. Schmahl and D. Rudolph for their continuous support and interest, and J. Thieme and B. Niemann for their contributions in the early part of this project. We also thank R. B. Hoover for assistance with diatoms, and $\mathrm{H}$. Brismar for assistance with fixed cells. This project was financed by the Swedish Engineering Science Research Council, the Swedish Natural Science Research Council, and the EC Human Capital and Mobility program.

\section{References}

Abraham-Peskir, J., Chantler, E., McCann, C., Medenwaldt, R. \& Ernst, E. (1998) Ultrastructure of human sperm using X-ray microscopy. Med. Sci. Research, 26, 663-667.

Aoki, S., Ogata, T., Iimura, K., Watanabe, N., Yoshidomi, Y., Shinada, K. \& Kato, T. (1998) A table-top grazing-incidence soft X-ray microscope with a laser-produced plasma source. X-Ray Microscopy and Spectromicroscopy (ed. by J. Thieme, G. Schmahl, D. Rudolph and E. Umbach), pp. I163-I167. Springer, Heidelberg.

Artioukov, I.A., Vinogradov, A.V., Asadchikov, V.E., Kas'yanov, Yu. S., Serov, R.V., Fedorenko, A.I., Kondratenko, V.V. \& Yulin, S.A. (1995) Schwarzschild soft x-ray microscope for imaging of nonradiating objects. Opt. Lett. 20, 2451-2453.

Berglund, M., Rymell, L. \& Hertz, H.M. (1996) Ultraviolet prepulse for enhanced x-ray emission and brightness from droplet-target laser plasmas. Appl. Phys. Lett. 69, 1683-1685.

Berglund, M., Rymell, L., Hertz, H.M. \& Wilhein, T. (1998) Cryogenic liquid-jet target for debris-free laser-plasma soft X-ray generation. Rev. Sci. Instrum. 69, 2361-2364.

Boese, J., Osanna, A., Jacobsen, C. \& Kirz, J. (1997) Carbon edge XANES spectroscopy of amino acids and peptides. J. Electron Spectrosc. Relat. Phenom. 85, 9-15.

Chapman, H.N., Jacobsen, C. \& Williams, S. (1996) A characterisation of dark-field imaging of colloidal gold labels in a scanning transmission X-ray microscope. Ultramicroscopy, 62, 191-213.

Da Silva, L.B., Trebes, J.E., Balhorn, R., Mrowka, S., Anderson, E., Attwood, D.T., Barbee, T.W., Brase, J., Corzett, M., Gray, J., Koch, J.A., Lee, C., Kern, D, London, R.A., MacGowan, B.J., Matthews, D.L. \& Stone, G. (1992) X-ray laser microscopy of rat sperm nuclei. Science, 258, 269-271.

Hertz, H.M., Rymell, L., Berglund, M. \& Malmqvist, L. (1998)
Debris-free liquid-target laser-plasma X-ray sources for microscopy and lithography. X-Ray Microscopy and Spectromicroscopy (ed. by J. Thieme, G. Schmahl, D. Rudolph and E. Umbach), pp. V3-V13. Springer, Heidelberg.

Hertz, H.M., Rymell, L., Berglund, M., Johansson, G.A., Wilhein, T., Platonov, Y. \& Broadway, D. (1999) A normal-incidence condenser mirror arrangement for compact water-window Xray microscopy. Proc. SPIE, 3766, 247-251.

Magowan, C., Brown, J.T., Liang, J., Heck, J., Coppel, R.L., Mohandas, N. \& Meyer-Ilse, W. (1997) Intracellular structures of normal and aberrant Plasmodium falciparum malaria parasites imaged by soft X-ray microscopy. Proc. Natl. Acad. Sci. 94, 62226227.

Nakayama, S., Haramura, K., Zeng, G.M., Daido, H., Nakatsuka, M., Nakai, S., Katakura, N., Nagata, H. \& Aritome, H. (1994) Zoneplate X-ray microscope using a laser plasma source. Jpn. J. Appl. Phys. 33, L1280-L1282.

Kirz, J., Jacobsen, C. \& Howells, M. (1995) Soft X-ray microscopes and their biological applications. Q. Rev. Biophys. 28, 33-130.

Moronne, M.M. (1999) Development of X-ray excitable luminescent probes for scanning X-ray microscopy. Ultramicroscopy, 77, 23-36.

Peuker, M., Schneider, G. \& Weiss, D. (1998) High resolution phase zone plates for water window wavelength. Proc. SPIE, 3449, $118-128$.

Rudolph, D., Schmahl, G., Niemann, B., Diehl, M., Thieme, J., Wilhein, T., David, C. \& Michelmann, K. (1994). X-Ray Microscopy IV (ed. by V. V. Aristov and A. I. Erko), pp. 381-386. Bogorodskii Pechatnik Publishers, Chernogolovka, Moscow.

Rymell, L. \& Hertz, H.M. (1993) Droplet target for low-debris laserplasma soft X-ray generation. Opt. Commun. 103, 105-110.

Rymell, L. \& Hertz, H.M. (1995) Debris-elimination in a droplettarget laser-plasma soft x-ray source. Rev. Sci. Instrum. 66, 4916-4920.

Rymell, L., Berglund, M. \& Hertz, H.M. (1995) Debris-free singleline laser plasma source for microscopy. Appl. Phys. Lett. 66, 2625-2627.

Salashchenko, N.N., Platonov, Y.u.Y.a., Z.u.e.v., S. \& Yu. (1995) Multilayer X-ray optics for synchrotron radiation. Nucl. Instrum. Meth. A, 359, 114-120.

Schmahl, G., Niemann, B., Rudolph, D., Diehl, M., Thieme, J., Neff, W., Holz, R., Lebert, R., Richter, F. \& Herziger, G. (1992) A laboratory X-ray microscope with a plasma X-ray source. X-Ray Microscopy III (ed. by A. G. Michette, G. R. Morrison and C. J. Buckley), pp. 66-74, Springer, Berlin.

Schmahl, G., Rudolph, D., Niemann, B., Guttmann, P., Thieme, J., Schneider, G., David, C., Diehl, M. \& Wilhein, T. (1993) X-ray microscopy studies. Optik, 93, 95-102.

Schneider, G. (1998) Cryo- X-ray microscopy with high spatial resolution in amplitude and phase contrast. Ultramicroscopy, 75 , 85-104.

Schneider, G., Schliebe, T. \& Aschoff, H. (1995) Cross-linked polymers for nanofabrication of high-resolution zone plates in nickel and germanium. J. Vac. Sci. Technol. B13 (6), 28092812.

Scherfeld, D., Schneider, G., Guttmann, P. \& Osborne, M. (1998) Visualization of cytoskeletal elements in the transmission X-ray microscope. J. Structural Biology, 123, 72-82. 
Stead, A.D., Ford, T.W., Myring, W.J. \& Clarke, D.T. (1988) A comparison of soft X-ray contact microscopy with light and electron microscopy for the study of algal cell structure. J. Microsc. 149, 207-216.

Thieme, J., Niemeyer, J., Machulla, G. \& Schulte-Ebbert, U. (1998) Aggregation of colloids observed by X-ray microscopy. X-Ray
Microscopy and Spectromicroscopy (ed. by J. Thieme, G. Schmahl, D. Rudolph and E. Umbach), pp. II11-II19. Springer, Heidelberg. Wilhein, T., Hambach, D., Niemann, B., Berglund, M., Rymell, L. \& Hertz, H.M. (1997) Off-axis reflecting zone plate for quantitative soft x-ray source characterization. Appl. Phys. Lett. 71, 190-192. 